Journal of Engineering and Applied Sciences 14 (4): 1178-1181, 2019

ISSN: 1816-949X

(C) Medwell Journals, 2019

\title{
Study of the Jet Reconstruction Algorithm Methods
}

\author{
Areeg Al-Hamadani and Ali Al-Dulaimi \\ College of Science, University of Diyala, Diyala, Baqubah, Iraq
}

\begin{abstract}
Jet reconstruction is the most important part in order to understand the way that the observed partons have to be linked as well as the jet partons harmonized to which particles. If the linking method has been understood, the properties of such partons can be known. In this study different algorithms that used to reconstruct jets will be presented and compared, $\mathrm{k}_{\mathrm{t}}$, Cambridge/Aachen and anti- $\mathrm{k}_{\mathrm{t}}$ Iterative cones in addition to the SISCone method, after the comparison found that is the best algorithm is the anti- $\mathrm{k}_{\mathrm{t}}$ algorithm whereas the Cambridge/Aachen method is the best way to study the substructures of jets.
\end{abstract}

$\underline{\text { Key words: Partons, jet linking, harmonized, transvers momentum, particles, reconstruct }}$

\section{INTRODUCTION}

A jet is a tighten cone of partons and other particles that produced in the process of the quarks or gluon harmonization after the collision. In order to connect the information that collected from the calorimeters and trackers, we use the jet algorithms to rebuilt jets. Studying the jets is important because it provides the information about the relation between the observed particles and standard model physics in the level of before particles have been hadronized. In the processes of proton proton collision, a shower of different particle results creates a jet as shown in Fig. 1. Linking methods gives wide information about the mother partons kinematics that helps to find more about properties of important particles as Higgs boson and other fast decaying particles. Once one chose the precise linking algorithm, the energy that misses in the detector can be calculated accurately leading to finding more in the beyond the standard model (Atkin, 2015).

There are some important points have to be taken into account before using the linking algorithms. First of all is the size and the area of the jet determine the capability of jets to soft radiation, the larger the jets are the more harmonized particles captured to better calculations for the mass and energy. In the other hand, the smaller jet area is good in the case of decreasing the Underlying Events amount (UE) and the Pileup (PU) that seized by the jet which bans recalculate the jet energy and mass. Second, the used algorithm is it the Infrared/Collinear (IRC) safe or not because if the not safe linking algorithm used, will not give a precise jet's contents and number but only in case of a soft gluon using the unsafe infrared algorithm will not affect the result (Salam and Soyez, 2007).

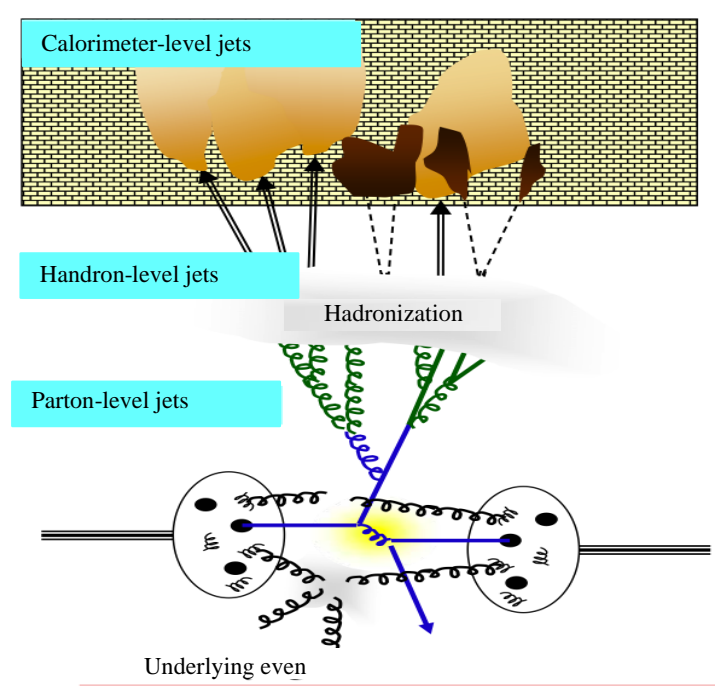

Fig. 1: An easy example of an event that show the collision point, then the fragmentation, the quarks and gluon hadronization process that make jet which is detected as stable particles (Salam and Soyez, 2007). Calojets, jets created by the calorimeter output also Genjets, jets created by stable simulated particles. The missing energy is repeated by the dashed line

\section{MATERIALS AND METHODS}

Jet algorithms: Jet algorithms are found in two kinds of the mainstream. The first kind is the Cone-type algorithms as iterative Cone (CMS) (Geoffrey et al., 1983), Midpoint Cone (Tev), SISCone (LHC). These algorithms are not infrared and collinear safe except the SISCone algorithm. Those algorithms are complex because they involve a lot of nonphysical parameters. It widely used in

Corresponding Author: T.A. Areeg Al-Hamadani, College of Science, University of Diyala, Diyala, Baqubah, Iraq 

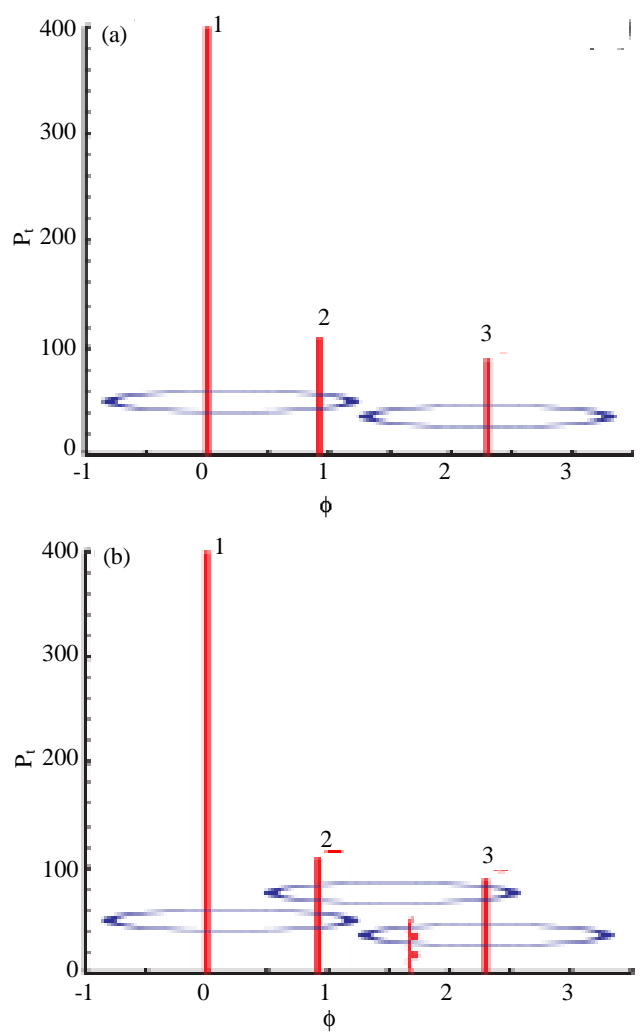

Fig. 2: Configuration illustrate one of the IR unsafe problems in the midpoint jet clustering algorithm $(\mathrm{R}=1)$ : a) A stable cones (shaped as ellipses) that found in the midpoint algorithm and b) With an addition of the arbitrarily soft velocity particle (the wavy red line) the extra stable cone is found (Coco et al., 2009).

computational hadron colliders. The second kind is the sequential clustering algorithms as Cambridge/Aachen (Cacciari et al., 2008a, b), anti- $\mathrm{k}_{\mathrm{t}}, \mathrm{k}_{\mathrm{t}}$ (Coco et al., 2009) algorithms which are infra-red and collinear safe, clean and not difficult to use.

Cone algorithms: In the cone reconstruction algorithm, the jet's particles appear in a conical region and the clustering method depends on the $(\eta-\varphi)$ space which makes the jet has circular confines and it is IRC unsafe. Because the cone algorithm contains some constant that not related to physics most theorists researchers don't choose to use it whereas experimentalists choose cone algorithm as it easy to perform.

Midpoint cone algorithm: In the midpoint algorithm a seed particles cluster particles into a cone if $\Delta R<R$ where $\mathrm{R}$ is the cone radius and iterate until stable cones which means when the axis coincides with the momentum sum
Table 1: Particles 1-3 represent a hard configuration. The jets from this hard configuration are modified in the midpoint cone algorithm when adds the soft particles are added (Salam and Soy ez 2007)

\begin{tabular}{llll}
\hline Particle & $\mathrm{p}_{\mathrm{t}}$ & $\mathrm{y}$ & $\Phi$ \\
\hline 1 & 400 & 0 & 0 \\
2 & 110 & $0.9 \mathrm{R}$ & 0 \\
3 & 90 & $2.3 \mathrm{R}$ & 0 \\
4 & 1.1 & $1.5 \mathrm{R}$ & 0 \\
\hline
\end{tabular}

then search for cones at the midpoint of cones at the midpoint of stable cones, jets merge if the overlapping energy is more than the energy of the smaller jet (Coco et al., 2009). From Table 1 configurations and Fig. 2 we can see that the problem of the midpoint algorithm is using 1-3 particles there three stable cones will exist. In the transverse momentum scheme $\mathrm{p}_{\mathrm{T}}$ a procedure for recombination they are at $\mathrm{y} \sim\{0.194,1.53$, $2.3 \mathrm{R}$ \} but starting from particles $1-3$ as seeds the iterative will be to the stable-cone at $\mathrm{y} \approx 0.194 \mathrm{R}$ and $\mathrm{y}=2.3 \mathrm{R}$. In another word, the problem is with finding all the stable cones.

Iterative cone linking algorithm with progressive removal IC-PR: It is one of the unsafe collinear algorithms as pythia cone and CMS iterative cone. In this algorithm at the first step find the cell with the largest transverse momentum $\mathrm{p}_{\mathrm{T}}$ and use it as a seed then around the chosen seed make a cone has a radius of $\mathrm{R}$, after that using the four vectors determine the axis for the suggested jet by adding up all the cells rounded by the chosen cone which is then tagged as stable and remove all the particles inside that cone from the list. After that, the other hardest $\mathrm{p}_{\mathrm{T}}$ cells are chosen and repeat the same steps. In some cases, the test jet axis won't match the seed axis, so, in this case, the jet axis will be the seed axis and redo all the steps until no more seeds left with energy higher than the threshold.

Iterative cone linking algorithm with the split merges IC-SM: In this kind of cone algorithm at the first step determine the threshold Energy E and make the cells all that have energy above the threshold cut the seed. Search for the stable cones that belong to those seeds and do the same steps in the IC-PR but without doing the step when removing the particles from the list after finding the stable cones. In this method when the stable cone been found it is labeled to project and run the procedure of splitting merge (Atkin, 2015) on that projects after finding them all. This algorithm is one of the infrared unsafe algorithms such as ATLAS cone, JetClu.

The seedless infrared safe cone linking algorithm SISCone: It is one of the cone's algorithms that's IRC safe has a comparatively small area which makes it not 
very affected by the underlying events and pile up with good resolution but it does not work very well with multi-jets. In the hard radiation case, SISCone algorithm can spread to the area bigger than $\mathrm{R}$ with high resolution which is not good at analyzing multi-jets. To use SISCone method to find jets we have to follow the steps below.

\section{SIScone algorithm:}

For the particle $\mathrm{i}$

Find the particles $j$ in the distance $2 R$ of $i$

If no particle $j$ are there

labeled $\mathrm{i}$ as stable cone then add it to the list of protojets Else

Find the circles that created by $\mathrm{i}$ and $\mathrm{j}$ lye on their circumference then calculate the

the cones momenta they define

For every circle

Treat the four replacements of both edge points to be counted inside or outside of the circle. The circles then are labeled as current cones

For every current cone that is not found before

Set if the current cones are inside or outside the edge status particles is same as the cone that defined using the momentum of the particles within the mentioned current cone. But if not have to label it as unstable

Check for stability on all the current cones which are not labeled as unstable then add stable cones all to the protojets list

Then on the protojets run a split merge step

Finally we can say that for SISCone algorithm it's clearly to see that for single-particle jets are regular whereas in case of composite jets, there are many different shapes.

\section{RESULTS AND DISCUSSION}

Sequential clustering algorithms: Is infrared and collinear safe algorithm by the constructions, it is also, clean and simple. It wasn't used a lot at the hadrons collider in the past but after resolving the computational performance and accessing the jet area problems it becomes the theorist best kind of algorithm (Cacciari et al., 2008a, b). In this kind of clustering algorithm particles in jets assume that particles have same transverse momentum differences and depend on this assumption the particles are collected, so, the jets are represented in the $(\eta-\varphi)$ space. Most of the sequential clustering algorithms have the same procedure. First determine the distance between the particles $i$ and $j$ where $\mathrm{d}_{\mathrm{ij}}$ here is:

$$
\mathrm{d}_{\mathrm{ij}}=\min \left(\mathrm{k}_{\mathrm{t}_{\mathrm{i}}}^{2 \mathrm{p}}, \mathrm{k}_{\mathrm{t}_{\mathrm{j}}}^{2 \mathrm{p}}\right) \frac{\Delta_{\mathrm{ij}}}{\mathrm{D}} \text { and } \Delta_{\mathrm{ij}}^{2}=\left(\mathrm{y}_{\mathrm{i}}-\mathrm{y}_{\mathrm{j}}\right)^{2}+\left(\phi_{\mathrm{i}}-\phi_{\mathrm{j}}\right)^{2}
$$

Then, determine the distance between the particle $\mathrm{i}$ and the beam that is $\mathrm{d}_{\mathrm{B}}=\mathrm{k}_{\mathrm{t}}^{2 \mathrm{p}}$ after calculate all the distances $\mathrm{d}_{\mathrm{ij}} \mathrm{d}_{\mathrm{i} B}$ determine the smallest distance, if $\mathrm{d}_{\mathrm{ij}}$ is the smallest combine the particle $i$ and $j$ and update the distance and continue to find the next smallest distance but $\mathrm{d}_{\mathrm{iB}}$ if is the smallest particle $\mathrm{i}$ consider as jet. Those steps have to repeat until all particles have been clustered to jets. There are three sequential clustering algorithm, $k_{t}$, antik $k_{t}$ Cambirdge/Aachen (C/A) which are differ than each other in the parameter (a) that determine the relative energy power verses the geometrical scale where it is equal to 2 in the $k_{b}-2$ in the antik $k_{t}$ and 0 in the C/A algorithm (Fig. 3).

The k: One of the oldest sequential algorithms which has $\mathrm{p}$ parameter that equal to 2 and the distance equation is:

$$
d_{i j}=\min \left(k_{t_{i}}^{2 p}, k_{t_{j}}^{2 p}\right) \frac{R_{i j}^{2}}{R}, d_{i B}=k_{t_{i}}^{2}
$$

The measured distance in this algorithm is exceedingly related to the divergences structure in the QCD emission. In the past was not used because it is slow computationally but after the fast jet implementation it became faster and it is work perfectly on subjets. $\mathrm{K}_{\mathrm{t}}$ algorithm first clusters the soft particles which make a fluctuated area which is oversensitive to the Underlying Events UE as well as the Pileup PU.

Cambridge/Aachen C/A: In this algorithm, the distance is measured depend on geometrical scale and the particles are grouped based on location, not energies or momentum. Cambridge/Aachen gives the best result in the jet substructure. The $\mathrm{p}$ parameter is equal to 0 in this algorithm and the distance equation will be:

$$
d_{i j}=\frac{R_{i j}^{2}}{R}, d_{i B}=1
$$

The distance variables are not depend on momentum which resulting a wiggly and oversensitive to the PU and $\mathrm{UE}$ and it is slightly more complicated than $\mathrm{k}_{\mathrm{t}}$ algorithm.

Antik: Is one of the jet finding algorithms that used in the hadron hadron collisions and one of the sequential reconstruction algorithms. It is IRC safe but it gives a shape of circular cone jet also it is not PU and UE sensitive. The parameter $\mathrm{p}$ in the anti- $\mathrm{k}_{\mathrm{t}}$ algorithm is equal to -2 and the distance equation is:

$$
\mathrm{d}_{\mathrm{ij}}=\min \left(\frac{1}{\mathrm{k}_{\mathrm{t}_{\mathrm{i}}}^{2}}, \frac{1}{\mathrm{k}_{\mathrm{t}_{\mathrm{j}}}^{2}}\right) \frac{\mathrm{R}_{\mathrm{ij}}^{2}}{\mathrm{R}}, \mathrm{d}_{\mathrm{iB}}=\frac{1}{\mathrm{k}_{\mathrm{t}_{\mathrm{i}}}^{2}}
$$

From the equation above one can see that it is controlled by the high transverse momentum and clustering at first the hardest particle. This algorithm is the best at jet resolving but it is worst at jet substructure because it is weak at the de-clustering. 

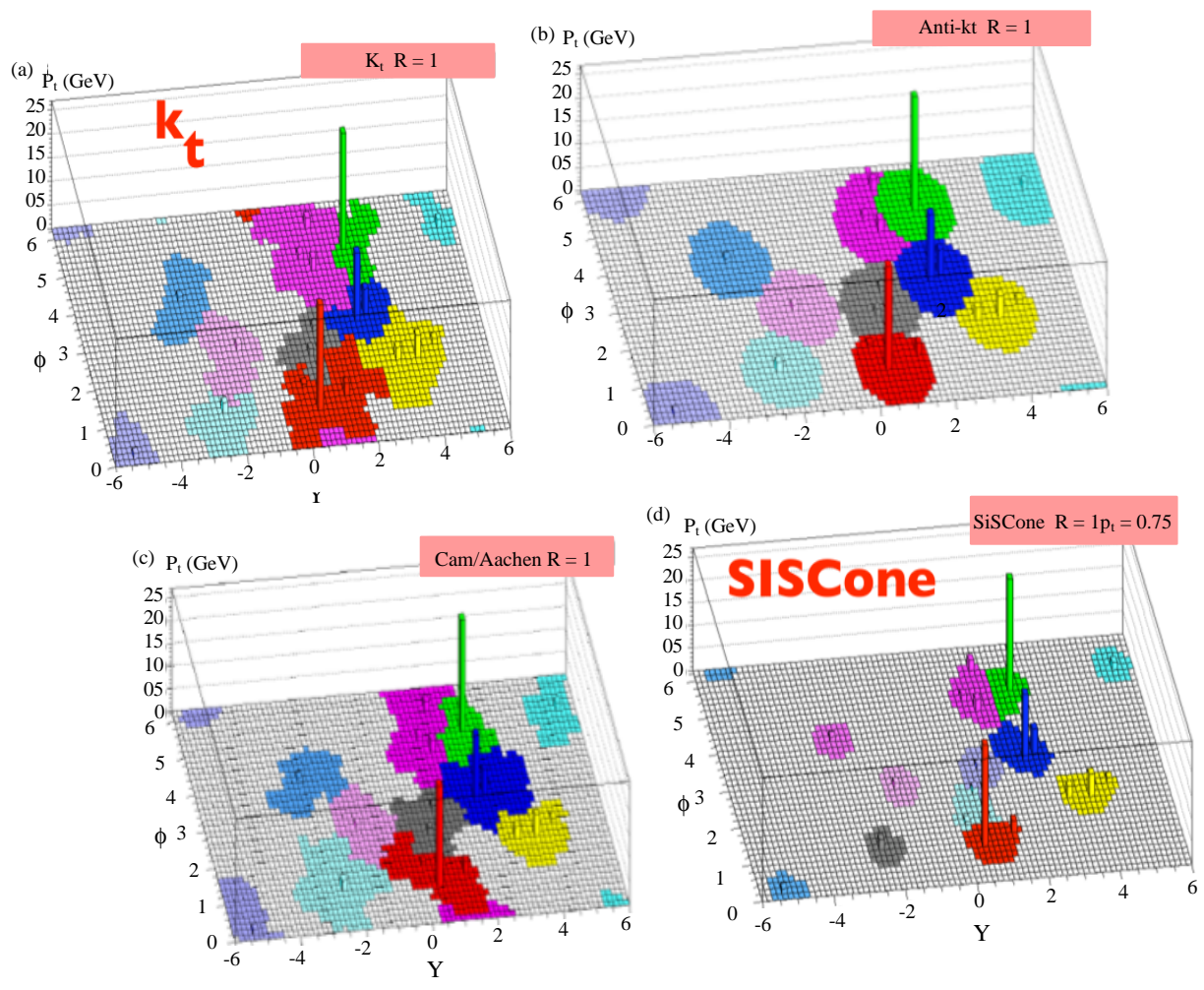

Fig. 3: The four most important clustering algorithms in the all cases the same data and same radius have been taken: a) $\mathrm{k}_{\mathrm{t}}$ algorithm; b) Anti- $\mathrm{k}_{\mathrm{t}}$ algorithm; c) CamLAachen algorithm and d) SISCone algorithm

\section{CONCLUSION}

Jets are the important key to study the resultant particles come from the hadron-hadron collision, to get better understanding have to choose the appropriate algorithm to reconstruct jets and to do that there are two things have to take into account, the infrared and collinear safety. As a result, of that researchers are not using the procedure of iterative cone with split merge and the iterative cone with progressive removal anymore. The other important parameters that have to take care of are the jet size and shape. The more jet captured the less underling and pileup effect. To study jets the more precise clustering algorithm is the anti- $\mathrm{k}_{\mathrm{t}}$ algorithm. On the other hand, to study the jet substructure the best to use is the Cambridge/Aachen algorithm. Finlay, the precise jet reconstruction algorithm is the method that helps us study the quarks and the gluons properties because we cannot observe them directly.

\section{ACKNOWLEDGEMENT}

The researchers acknowledge their family and every one support them emotionally and practically.

\section{REFERENCES}

Atkin, R., 2015. Review of jet reconstruction algorithms. J. Phys. Conf. Ser., 645: 1-7.

Cacciari, M., J. Rojo, G.P. Salam and G. Soyez, 2008b. Quantifying the performance of jet definitions for kinematic reconstruction at the LHC. J. High Energy Phys., 2008b: 1-24.

Cacciari, M., S. Frixione, M.L. Mangano, P. Nason and G. Ridolfi, 2008a. Updated predictions for the total production cross sections of top and of heavier quark pairs at the Tevatron and at the LHC. J. High Energy Phys., 2008: 1-19.

Coco, V., P.A. Delsart, J. Rojo and C.S.G. Soyez, 2009. Jets and jet algorithms. J. Eng. Sci., 1: 182-204.

Geoffrey, T.J.A., A. Alan, A. Bernard, B. Cesare and C. Roberts et al., 1983. Hadronic jet production at the CERN proton-antiproton collider. Phys. Lett. B, 132: 214-222.

Salam, G.P. and G. Soyez, 2007. A practical seedless infrared-safe cone jet algorithm. J. High Energy Phys., 2007: 1-42. 